\title{
La metformina en mujeres embarazadas obesas sin diabetes no redujo el peso al nacimiento del recién nacido
}

\author{
Metformin in obese pregnant women without diabetes did not reduce birth weight of the newborn
}

\section{Comentado de:}

Dodd JM et al. Cochrane Database Syst Rev. 2018 Jul 24;7:CD010564 ${ }^{1}$

\section{Objetivos}

Evaluar la función de la metformina en las pacientes embarazadas con obesidad o que presentan sobrepeso sobre los resultados maternos e infantiles, incluidos los efectos adversos del tratamiento y los costos.

\section{Métodos de búsqueda}

Se hicieron búsquedas en el registro de ensayos del Grupo Cochrane de Embarazo y Parto, ClinicalTrials.gov, la World Health Organization (WHO) International Clinical Trials Registry Platform (ICTRP) (hasta octubre de 2017), y en las listas de referencia de los estudios recuperados.

\section{Criterios de selección}

Fueron elegibles para inclusión todos los ensayos controlados aleatorizados, publicados y no publicados, que evaluaron la administración de metformina en pacientes con obesidad o que presentaban sobrepeso durante el embarazo, sola o en combinación con otras intervenciones, en comparación con placebo o ningun otro tratamiento farmacológico.

\section{Obtención y análisis de los datos}

Dos revisores evaluaron de forma independiente los ensayos para la inclusión y el riesgo de sesgo, extrajeron los datos y verificaron su exactitud. Se utilizó el enfoque GRADE para evaluar la calidad de la evidencia.

\section{Resultados principales}

Se incluyeron tres estudios que asignaron al azar a 1.099 pacientes con un índice de masa corporal (IMC) de $30 \mathrm{~kg} / \mathrm{m}^{2}$ (un estudio) y $35 \mathrm{~kg} / \mathrm{m}^{2}$ (dos estudios), con resultados disponibles para 1.034 participantes. Ninguno de los estudios evaluó pacientes con un IMC entre $25 \mathrm{~kg} / \mathrm{m}^{2}$ y $29,9 \mathrm{~kg} / \mathrm{m}^{2}$, por lo que no fue posible evaluar la administración de metformina en las pacientes con sobrepeso. No se identificaron estudios de metformina en combinación con otro tratamiento. Otros dos estudios están en curso.

Los tres estudios incluidos fueron ensayos aleatorizados y controlados que compararon metformina con placebo, que se iniciaron a principios del segundo trimestre de gestación. Las dosis variaron entre $500 \mathrm{mg}$ dos veces al día y $3 \mathrm{~g}$ por día. Los tres estudios (dos en el Reino Unido, uno en Egipto) incluyeron pacientes que asistían a hospitales para atención prenatal.

Dos estudios tuvieron un riesgo general de sesgo bajo en la mayoría de los dominios. El tercer estudio se evaluó como riesgo incierto de sesgo de selección, realización y detección debido a que la información fue insuficiente, como bajo riesgo de sesgo de desgaste y con alto riesgo de sesgo de reporte.

El resultado primario para esta revisión fue peso del recién nacido $(\mathrm{RN})$ grande para la edad gestacional al parto (percentilo $>90$ para la edad gestacional y el sexo del RN). Las pacientes que recibieron metformina o placebo tuvieron un riesgo similar de tener un parto de un RN grande para la edad gestacional al parto (cociente de riesgos [CR] 0,95; intervalo de confianza [IC] del
$95 \%$ : 0,70 a 1,30; dos estudios, 831 lactantes; evidencia de alta calidad).

Las pacientes que recibieron metformina pueden tener un aumento de peso gestacional algo inferior (diferencia de medias [DM] $-2,60 \mathrm{~kg}$, IC del $95 \%$ : -5,29 a 0,10; tres estudios, 899 mujeres; evidencia de baja calidad).

La metformina puede lograr poco o ningún cambio en el riesgo de las pacientes de desarrollar hipertensión gestacional (CR promedio 1,02; IC 95\%: 0,54 a 1,94; tres estudios, 1040 mujeres; evidencia de baja calidad), preeclampsia (CR 0,74; IC $95 \%$ : 0,09 a 6,28; dos estudios, 840 mujeres; evidencia de baja calidad) o diabetes gestacional (CR 0,85; IC $95 \%$ : 0,61 a 1,19; tres estudios, 892 mujeres; evidencia de calidad moderada).

Un estudio de 400 mujeres informó que las pacientes que recibieron metformina tuvieron mayores probabilidades de presentar cualquier efecto adverso en comparación con las pacientes que recibieron placebo (CR 1,63; IC 95\%: 1,27 a 2,08). Los efectos adversos incluyeron dolor abdominal, diarrea o cefalea. Cuando se consideraron los efectos secundarios individuales, las pacientes que recibieron metformina tuvieron mayores probabilidades de presentar diarrea que las pacientes que recibieron placebo (CR 2,34; IC del 95\%: 1,74 a 3,14; 797 mujeres; dos estudios, 797 mujeres; evidencia de alta calidad). No se identificaron otras diferencias importantes entre metformina y placebo para otros resultados secundarios maternos, incluidos: parto por cesárea, parto antes de las 37 semanas de embarazo, distocia de hombro, desgarro perineal o hemorragia postparto.

En cuanto a otros resultados infantiles, hubo poco o ningún cambio en el peso del recién nacido al parto (DM 6,39 g; IC 95\%: $-81,15$ a 93,92; dos estudios, 834 lactantes; evidencia de alta calidad). No se identificaron otras diferencias importantes para otros resultados secundarios del lactante en esta revisión: hipoglucemia, ictericia, puntuación de Apgar menor de 7 a los cinco minutos, o mortinatalidad y muerte neonatal. Solo un estudio informó el ingreso a la unidad de cuidados intensivos neonatales (UCIN), con tasas similares de ingreso entre las pacientes que recibieron metformina o placebo; no se informaron otros datos del ingreso para evaluar las diferencias en los costos.

\section{Conclusiones de los autores}

No hay evidencia suficiente para apoyar la administración de metformina en las pacientes con obesidad durante el embarazo para mejorar los resultados maternos e infantiles. Sin embargo, la metformina se asoció con un mayor riesgo de efectos adversos, en particular de diarrea. La principales causas para disminuir la calidad de la evidencia en esta revisión fueron las limitaciones de los estudios y la inconsistencia. Sin embargo, hubo un escaso número de estudios, ninguno de ellos incluyó pacientes categorizadas como sobrepeso ni examinó la metformina en combinación con otro tratamiento.

Se necesitan más estudios para evaluar la función del tratamiento con metformina en las pacientes embarazadas con obesidad o que presentan sobrepeso como una estrategia para mejorar la salud materna e infantil, ya sea sola o como coadyuvante a la asesoría dietética o sobre el estilo de vida. 


\section{Comentario}

La obesidad materna se asocia con resultados perinatales adversos y es un factor de riesgo independiente para la hiperinsulinemia fetal, el peso al nacimiento y la adiposidad del recién nacido, además de asociarse con obesidad infantil y obesidad en la vida adulta. Como lo confirman los hallazgos de la revisión resumida, la metformina mejora la sensibilidad a la insulina y que en mujeres embarazadas con diabetes gestacional conduce a una menor ganancia de peso durante el embarazo. Se ha observado también que la obesidad y el síndrome metabólico se asocian a inflamación sistémica y aumento de la morbilidad perinatal, y la metformina, en modelos in vivo e in vitro, logró reducir los niveles de citoquinas maternas y fetales, mejorando la inflamación ${ }^{2}$. Sin embargo, el análisis de la evidencia proveniente de ensayos con pacientes no ha podido demostrar que la metformina en embarazadas obesas no diabéticas haya mejorado el peso al nacimiento del recién nacido. A pesar de que un estudio previo había documentado una notable la reducción prácticamente del $80 \%$ en la incidencia de preeclampsia ${ }^{3}$, este beneficio no se confirmó al realizar el meta-análisis.

\section{Conclusiones de la comentadora}

Si bien la metformina es una medicación que no ha demostrado riesgo en humanos (categoría B) y podría tener un efecto benéfico sobre el riesgo futuro de obesidad y síndrome metabólico en los hijos nacidos de madres obesas, no se han documentado beneficios en los desenlaces perinatales y aún falta evidencia a largo plazo del seguimiento de aquellos niños expuestos al tratamiento con metformina intraútero.

María Fernanda Lage [ Servicio de Obstetricia, Hospital italiano de Buenos Aires maria.lage@hospitalitaliano.org.ar ]

Lage MF La metformina en mujeres embarazadas obesas sin diabetes no redujo el peso al nacimiento del recién nacido . Evid Actual Pract Ambul. 2019;22(2):. Comentado de: Dodd JM et al. Metformin for women who are overweight or obese during pregnancy for improving maternal and infant outcomes. Cochrane Database Syst Rev. 2018 Jul 24;7:CD010564. PMID 30039871

\section{Referencias}

1. Dodd JM, Grivell RM, Deussen AR, Hague WM. Metformin for women who are overweight or obese during pregnancy for improving maternal and infant outcomes. Cochrane Database of Systematic Reviews. 2018;2018. Available from: 10.1002/14651858.CD010564.pub2.

2. Desai N, Roman A, Rochelson B, Gupta M, Xue X, Chatterjee PK, et al. Maternal metformin treatment decreases fetal inflammation in a rat model of obesity and metabolic syndrome. American journal of obstetrics and gynecology. 2013;209(2). Available from: 10.1016/j.ajog.2013.05.001.

3. Syngelaki A, Nicolaides KH, Balani J, Hyer S, Akolekar R, Kotecha R, et al. Metformin versus Placebo in Obese Pregnant Women without Diabetes Mellitus. The New England journal of medicine. 2016 feb;374(5):434-43. Available from: 10.1056/NEJMoa1509819. 